\section{Case Reports in Dermatology}

Case Rep Dermatol 2017;9:1-7

\title{
Overlapping DRESS and Stevens- Johnson Syndrome: Case Report and Review of the Literature
}

\author{
Aneline Casagranda Mariano Suppa Florence Dehavay \\ Véronique del Marmol \\ Department of Dermatology and Venereology, Erasme Hospital, ULB, Brussels, Belgium
}

\section{Keywords}

Severe cutaneous adverse reactions · DRESS · Stevens-Johnson syndrome · RegiSCAR

\begin{abstract}
Drug-induced severe cutaneous adverse reactions (SCARs) include acute generalized exanthematous pustulosis, drug reaction with eosinophilia and systemic symptoms (DRESS), and epidermal necrolysis (Stevens-Johnson syndrome [SJS], toxic epidermal necrolysis). The identification of the causal drug is crucial in order to avoid further exposure, but making the right differential diagnosis of the type of SCAR is equally important since treatment, follow-up, and prognosis of different SCARs are not the same. These syndromes are distinct entities with different clinical, biological, and histological patterns, but sometimes the early distinction between 2 SCARs can be extremely challenging, and overlapping conditions could therefore be taken into consideration, although true overlapping SCARs are very rare when using strict diagnostic criteria (described by the RegiSCAR group). Only a better understanding of the physiopathology of the SCARs could possibly explain these ambiguities and overlaps. We report a case of SCAR in an 86-year-old patient probably induced by allopurinol and simultaneously fulfilling the diagnostic criteria for DRESS and SJS, thus considered as an overlapping case of SCARs.




\section{Case Reports in Dermatology}

\section{Introduction}

Severe cutaneous adverse reactions (SCARs) to drugs consist of different syndromes, including acute generalized exanthematous pustulosis (AGEP), drug reaction with eosinophilia and systemic symptoms (DRESS), and epidermal necrolysis (Stevens-Johnson syndrome [SJS], toxic epidermal necrolysis [TEN]). The differential diagnosis between these entities is usually clear because of their distinct clinical, biological, and histopathological characteristics.

AGEP is characterized by an acute onset of erythematous areas studded with small, nonfollicular, sterile pustules, initially in intertriginous areas, usually occurring $48-72 \mathrm{~h}$ after the introduction of the drug. Fever and neutrophilia are frequent [1]. Organ involvement is unusual and the evolution is usually favorable after drug discontinuation with a resolution in 2 weeks and a mortality rate of $2 \%$, especially in elderly patients with comorbidities [2].

DRESS is a drug reaction occurring about 2-6 weeks after the introduction of the causative drug. It presents as a pruriginous maculopapular rash (usually starting on the face and then generalized), with edema of the face and extremities, fever, and peripheral lymphadenopathies. Mucosal involvement is unusual, but systemic involvement - particularly at the expense of the liver and kidneys - is nearly always present and responsible for a mortality rate of $5 \%[1,3,4]$. Laboratory changes are marked by high eosinophil count, atypical lymphocytes, and possibly elevated liver enzymes and/or decreased glomerular filtration rate (GFR) [1, 3]. Reactivation of the herpes virus family, especially human herpes virus 6 (HHV6), can be found [5]. Evolution is characterized by frequent flare-ups and autoimmune complications such as thyroiditis, diabetes, or lupus, thus requiring a close follow-up $[3,6]$.

Epidermal necrolysis is characterized by the occurrence (4 days to 4 weeks after the introduction of the drug) of a flu-like syndrome followed by a maculopapular rash, with blistering and skin detachment (positive Nikolsky sign) [1]. The surface of the skin detachment categorizes this SCAR as SJS $(<10 \%)$, SJS/TEN overlap (10-30\%), or TEN, also called Lyell syndrome $(>30 \%)$. Mucous erosions are nearly always present, mostly on the oropharyngeal and ocular mucosae, and are responsible for major functional impairment [7]. The patient's general condition is usually altered, and there is a high risk of secondary bacterial infection and sepsis. Biological routine can show cytopenia and elevated liver/pancreatic enzymes, but eosinophilia is very rare [4, 7]. SJS and TEN are characterized by a high mortality rate, varying from 3 to $90 \%$ according to the Score for Toxic Epidermal Necrolysis (SCORTEN) [8, 9]. Sequelae or lasting disability are frequent, especially due to ocular mucosae involvement.

Sometimes these entities can share some features, raising the hypothesis of overlap syndromes [10]. Validation scores have been established by the "European Registry of SCARs to Drugs and Collection of Biological Samples" (RegiSCAR) group in order to retrospectively classify cases of SCARs as definite, probable, possible, or excluded $[2,11]$. An overlap can be considered when a patient fulfills the criteria (probable or definite case) for at least 2 SCARs. We report here a case of overlapping SCAR, meeting the RegiSCAR criteria for both DRESS and SJS.

\section{Case Report}

An 86-year-old woman, hospitalized for a urinary tract infection, presented a maculopapular rash, starting on the face and then extending to the trunk. She also had fever up to $40^{\circ} \mathrm{C}$. The mucosae were not involved, there was no skin detachment, and the Nikolsky sign 
was negative. The laboratory routine was normal, except for GFR $52 \mathrm{~mL} / \mathrm{min} / 1.72 \mathrm{~m}^{2}$, likely due to her known chronic renal failure. New drugs had been introduced 1 month earlier: levofloxacin (for 1 week only), allopurinol, bumetanide, and repaglinide (as chronic treatments). The initially proposed diagnosis was maculopapular exanthema due to allopurinol, which was thus stopped, and treatment by cetirizine $10 \mathrm{mg} /$ day and topical betamethasone valerate $0.1 \%$ twice daily was then initiated.

One week later, the rash had spread to the whole body, showing a reticular aspect (Fig. 1), with skin detachment on the knees and a positive Nikolsky sign only on this location. The patient also presented mucosal involvement on the lips, uvula, nose, and conjunctiva (Fig. 2).

The blood test showed major hypereosinophilia up to $5,000 / \mathrm{mm}^{3}$, worsening of the renal failure (GFR $27 \mathrm{~mL} / \mathrm{min} / 1.72 \mathrm{~m}^{2}$ ), and lightly increased $\gamma$-glutamyl transferase at $48 \mathrm{U} / \mathrm{L}$ (normal 5-36 U/L). Serology for HHV-6 was negative. A biopsy was taken from the left forearm. The histopathological examination revealed a necrotic epidermis with spongiosis, necrotic keratinocytes, and rare eosinophils, compatible with SJS (Fig. 3).

The patient was admitted and received oral methylprednisolone (32 mg/day) and a supportive local treatment for the mucosal involvement. The other new medications (bumetanide and repaglinide) were also stopped at this stage.

Within 4 days, we observed a significant improvement of the patient's condition: the rash decreased and the eosinophilia rate as well as the renal function returned to normal. Corticosteroids were maintained for several weeks with a very low decreasing dose, due to several flare-ups of the eosinophilic count during the treatment.

One month later, she presented with immune thrombocytopenic purpura, with a very low platelet count (down to $28,000 / \mathrm{mm}^{3}$ ), which was successfully treated by dexamethasone. She then developed deep vein thrombosis and a pulmonary embolism concomitant with pneumonia, which resolved in a couple of days. Further investigations using patch tests to confirm the suspect causal drug (allopurinol) were not performed because of the patient's poor general condition.

According to the RegiSCAR criteria [2,11], the clinical picture of our patient could be classified as "probable" both for DRESS and SJS. Therefore, our final diagnosis was overlapping DRESS/SJS.

\section{Discussion}

Although cutaneous reactions to systemic drugs represent a frequent condition - they can affect up to $3 \%$ of all hospitalized patients - they are considered severe only in a small proportion $(1-2 \%)$ of cases $[4,10]$, which are called SCARs. The differential diagnosis between the different types of SCARs - AGEP, DRESS, and SJS/TEN - is usually easy since these conditions frequently have a distinctive, typical presentation. In rare cases, though, the initial presentation of SCAR can be ambiguous, thus making the differential diagnosis more challenging. In some instances, this can lead to the suspicion of overlapping SCARs.

The RegiSCAR group established validation scores, using clinical/biological/histological parameters, in order to retrospectively classify cases as a "definite," "probable," "possible," or "excluded" diagnosis of AGEP, DRESS, or SJS/TEN [2,11]. A true overlap is considered when the case of a patient can be classified as "probable" or "definite" for 2 SCARs at the same time.

In the case of our patient, the clinical presentation was not entirely typical of a single particular SCAR. The eosinophilia, the long-term evolution with flare-ups, and the presence 
of an autoimmune complication (immune thrombocytopenic purpura) would suggest the diagnosis of DRESS. On the other hand, the mucosal involvement and the histopathological examination would be more consistent with the diagnosis of SJS. According to the available RegiSCAR scores, the patient fulfilled the criteria for a "probable" DRESS and also for a "probable" SJS, thus her case could be regarded as an overlapping DRESS/SJS. The causal drug could not be identified with certainty; however the most suspected drug was allopurinol. This drug was indeed described as involved in a large proportion of cutaneous drug reactions and more specifically in SCARs [12].

Only a few cases of overlapping SCARs have been reported, with all combinations being possible $[13,14]$. In the retrospective monocentric study of Bouvresse et al. [10], which included 216 cases of SCARs, ambiguities between the SCARs were frequent (21\%), but only 3 cases $(2.1 \%)$ of true overlap were found when applying the RegiSCAR validation scores.

The existence of overlapping cases can be explained at least in part by looking at the pathophysiology of the SCARs, although it has yet to be understood in its entirety. The 3 entities - AGEP, DRESS, and SJS/TEN - are categorized as delayed hypersensitivity reactions (type IV reactions according to the classification by Coombs and Gell) mediated by T cells [1, 5], which can activate different inflammatory cells and chemokines, thus leading to different clinical patterns. Some authors even subclassified delayed hypersensitivity reactions in 4 subgroups (IV a, b, c, d), depending on the chemokines produced and the cells activated. Certain alleles of human leukocyte antigens (HLA) are also involved in the activation of T cells, leading to these different activation pathways [1, 7]. For instance, the presence of HLAB*1502 was associated with a risk of carbamazepine-induced SJS/TEN in Vietnamese, HanChinese, Thai, and Malaysian populations [15]. One pathway is usually dominant, leading to a specific clinical expression, but sometimes these immune reactions can overlap or be combined, explaining ambiguities or overlapping cases.

When SCAR is suspected, not only is it important to identify the causal drug - in order for the patient to avoid further exposure to it - but it is also crucial to formulate a precise diagnosis, as different types of SCAR present huge differences in terms of treatment, prognosis, and follow-up. For instance, AGEP resolves usually within 2 weeks with no sequelae, whereas DRESS is characterized by long-term flare-ups and autoimmune complications, and SJS/TEN by a high mortality rate and long-term functional and esthetic sequelae.

In conclusion, we report here a case of an overlapping DRESS/SJS, probably due to allopurinol, which was formally diagnosed by means of the RegiSCAR validation scores. Our case highlights the importance of using strict diagnostic criteria when suspecting AGEP, DRESS, or SJS/TEN because of the differences in terms of treatment, follow-up, and short/long-term prognosis that characterize the different SCARs.

\section{Statement of Ethics}

Informed consent was obtained from the patient prior to publication.

\section{Disclosure Statement}

The authors declare no conflicts of interest. 


\section{Case Reports in Dermatology}

\section{References}

1 Paulmann M, Mockenhaupt M: Severe drug-induced skin reactions: clinical features, diagnosis, etiology, and therapy. J Dtsch Dermatol Ges 2015;13:625-645.

-2 Sidoroff A, Halevy S, Bavinck JN, Vaillant L, Roujeau JC: Acute generalized exanthematous pustulosis (AGEP) - a clinical reaction pattern. J Cutan Pathol 2001;28:113-119.

-3 Husain Z, Reddy BY, Schwartz RA: DRESS syndrome. Part I. Clinical perspectives. J Am Acad Dermatol 2013;68:693.e1-e14; quiz 706-708.

4 Wolf R, Orion E, Marcos B, Matz H: Life-threatening acute adverse cutaneous drug reactions. Clin Dermatol 2005;23:171-181.

5 Torres MJ, Mayorga C, Blanca M: Nonimmediate allergic reactions induced by drugs: pathogenesis and diagnostic tests. J Investig Allergol Clin Immunol 2009;19:80-90.

-6 Descamps V, Ben Saïd B, Sassolas B, Truchetet F, Avenel-Audran M, Girardin P, et al: Management of drug reaction with eosinophilia and systemic symptoms (DRESS) (in French). Ann Dermatol Venereol 2010;137:703-708.

-7 Schwartz RA, McDonough PH, Lee BW: Toxic epidermal necrolysis. Part I. Introduction, history, classification, clinical features, systemic manifestations, etiology, and immunopathogenesis. J Am Acad Dermatol 2013;69:173.e1-e13; quiz 185-186.

8 Schwartz RA, McDonough PH, Lee BW: Toxic epidermal necrolysis. Part II. Prognosis, sequelae, diagnosis, differential diagnosis, prevention, and treatment. J Am Acad Dermatol 2013;69:187.e1-e16; quiz 203-204.

-9 Bastuji-Garin S, Fouchard N, Bertocchi M, Roujeau JC, Revuz J, Wolkenstein P: SCORTEN: a severity-ofillness score for toxic epidermal necrolysis. J Invest Dermatol 2000;115:149-153.

10 Bouvresse S, Valeyrie-Allanore L, Ortonne N, Konstantinou MP, Kardaun SH, Bagot M, et al: Toxic epidermal necrolysis, DRESS, AGEP: do overlap cases exist? Orphanet J Rare Dis 2012;7:72.

-11 Kardaun SH, Sidoroff A, Valeyrie-Allanore L, Halevy S, Davidovici BB, Mockenhaupt M, et al: Variability in the clinical pattern of cutaneous side effects of drugs with systemic symptoms: does a DRESS syndrome really exist? Br J Dermatol 2007;156:609-611.

-12 Halevy S, Ghislain P-D, Mockenhaupt M, Fagot J-P, Bouwes Bavinck JN, Sidoroff A, et al: Allopurinol is the most common cause of Stevens-Johnson syndrome and toxic epidermal necrolysis in Europe and Israel. J Am Acad Dermatol 2008;58:25-32.

13 Kim TI, Jeong KH, Shin MK, Kim NI: Piperacillin/tazobactam-associated hypersensitivity syndrome with overlapping features of acute generalized exanthematous pustulosis and drug-related rash with eosinophilia and systemic symptoms syndrome. Ann Dermatol 2016;28:98-101.

14 González-Ramos J, Noguera-Morel L, Tong HY, Ramírez E, Ruiz-Bravo E, Bellón T, et al: Two cases of overlap severe cutaneous adverse reactions to benznidazole treatment for asymptomatic Chagas disease in a nonendemic country. Br J Dermatol 2016;175:604-607.

-15 Tangamornsuksan W, Chaiyakunapruk N, Somkrua R, Lohitnavy M, Tassaneeyakul W: Relationship between the HLA-B*1502 allele and carbamazepine-induced Stevens-Johnson syndrome and toxic epidermal necrolysis: a systematic review and meta-analysis. JAMA Dermatol 2013;149:1025-1032. 


\section{Case Reports in Dermatology}

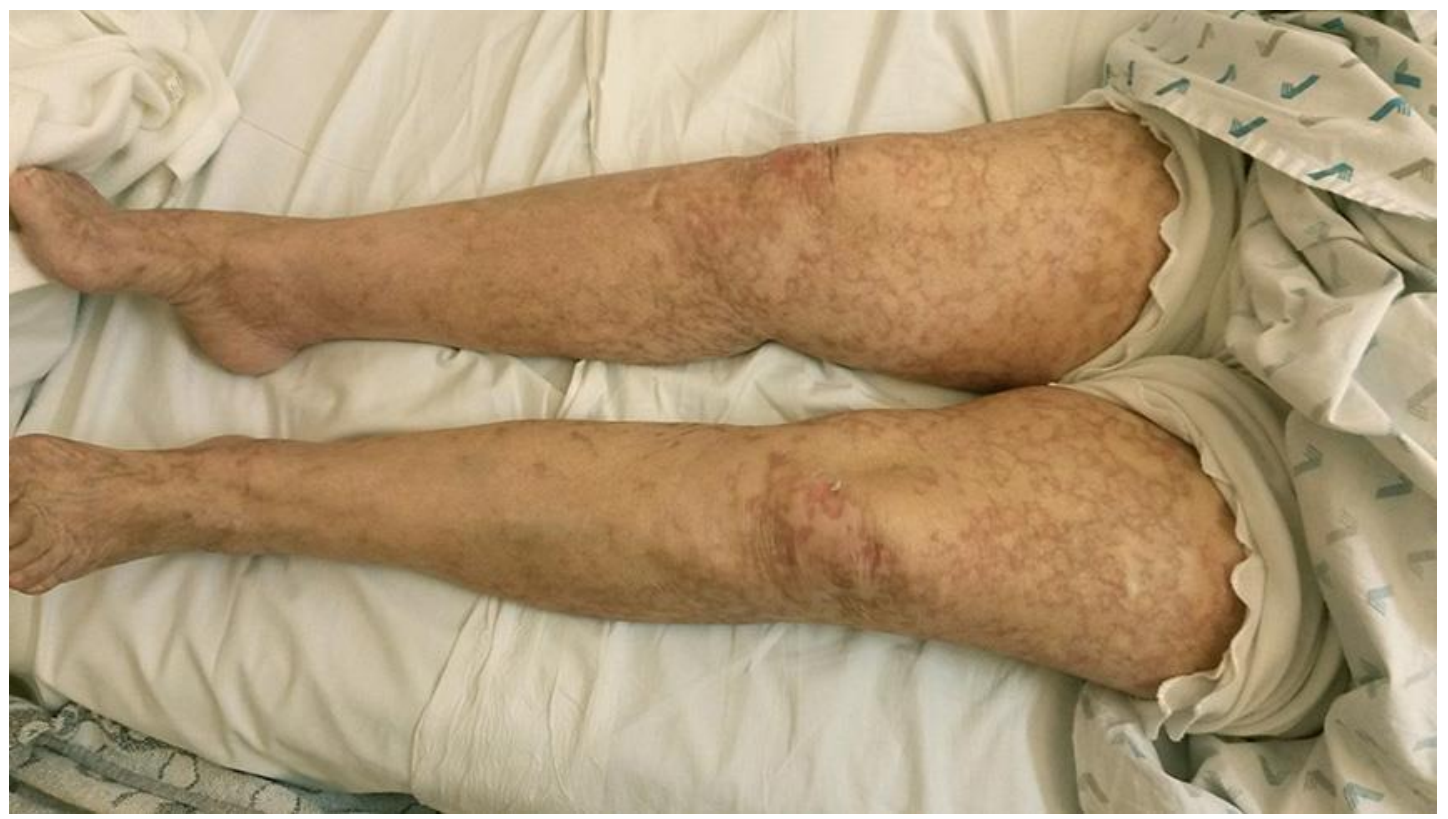

Fig. 1. Generalized rash showing a reticular aspect.

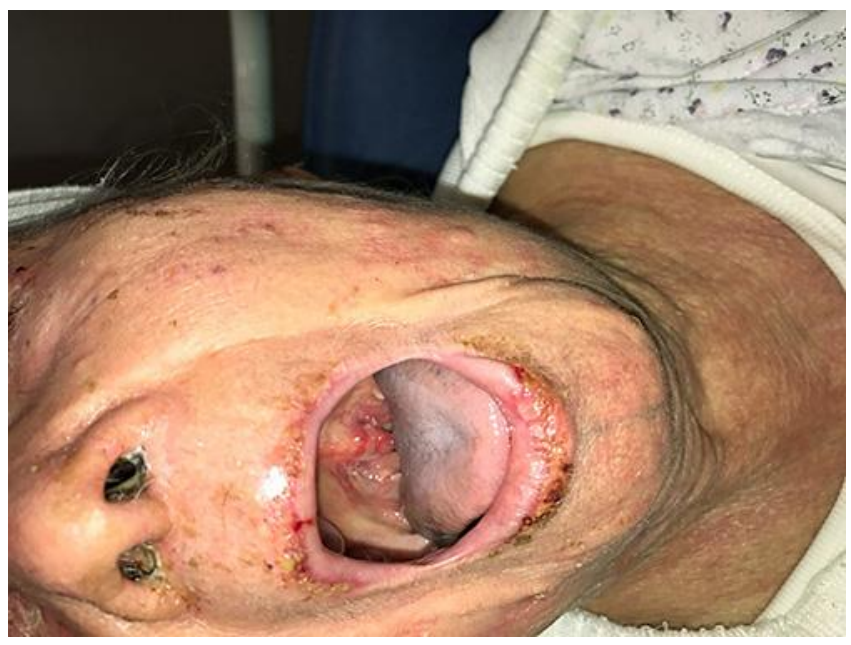

Fig. 2. Mucosal erosions involving lips and uvula. 


\section{Case Reports in Dermatology}

www.karger.com/cde

Casagranda et al.: Overlapping DRESS and Stevens-Johnson Syndrome: Case Report and Review of the Literature

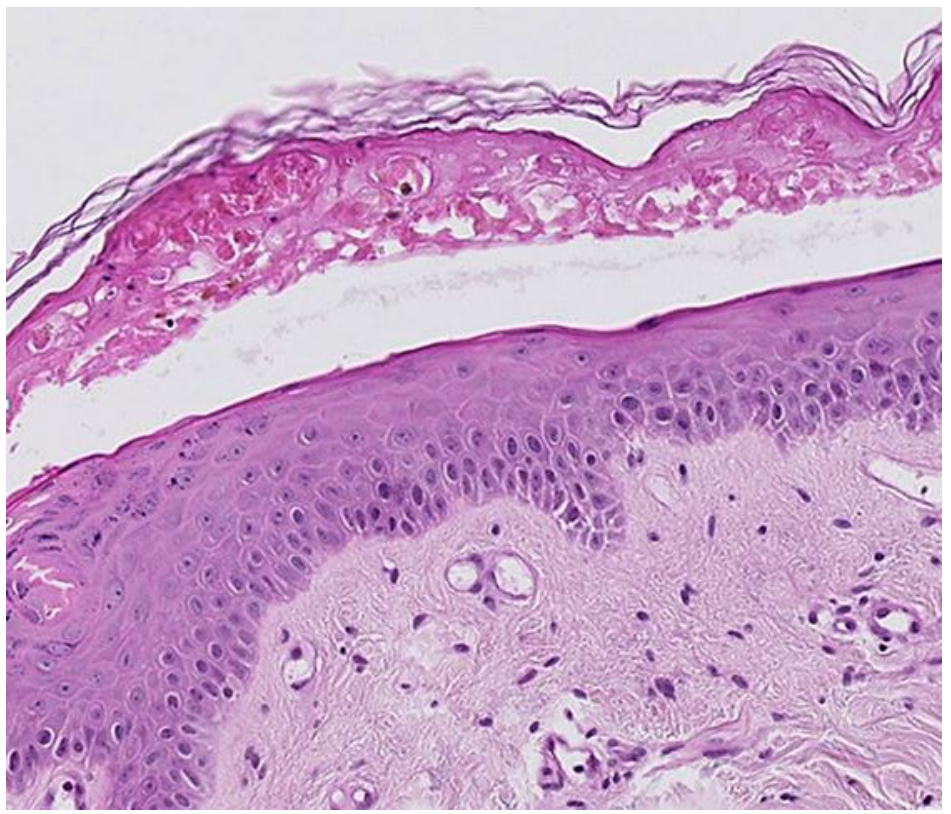

Fig. 3. Histopathology showed necrotic epidermis, with spongiosis and necrotic keratinocytes. H\&E. $\times 12$. 